\title{
Species and genus level resolution analysis of gut microbiota in Clostridium difficile patients following fecal microbiota transplantation
}

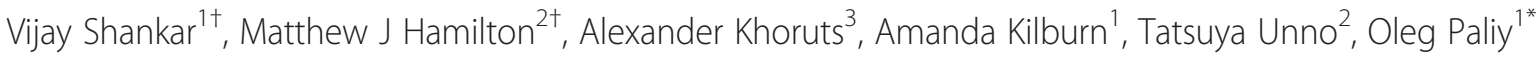 \\ and Michael J Sadowsky ${ }^{2^{*}}$
}

\begin{abstract}
Background: Clostridium difficile is an opportunistic human intestinal pathogen, and C. difficile infection (CDI) is one of the main causes of antibiotic-induced diarrhea and colitis. One successful approach to combat CDI, particularly recurrent form of $\mathrm{CDI}$, is through transplantation of fecal microbiota from a healthy donor to the infected patient. In this study we investigated the distal gut microbial communities of three CDI patients before and after fecal microbiota transplantation, and we compared these communities to the composition of the donor's fecal microbiota. We utilized phylogenetic Microbiota Array, high-throughput Illumina sequencing, and fluorescent in situ hybridization to profile microbiota composition down to the genus and species level resolution.
\end{abstract}

Results: The original patients' microbiota had low diversity, was dominated by members of Gammaproteobacteria and Bacilli, and had low numbers of Clostridia and Bacteroidia. At the genus level, fecal samples of CDI patients were rich in members of the Lactobacillus, Streptococcus, and Enterobacter genera. In comparison, the donor community was dominated by Clostridia and had significantly higher diversity and evenness. The patients' distal gut communities were completely transformed within 3 days following fecal transplantation, and these communities remained stable in each patient for at least 4 months. Despite compositional differences among recipients' pre-treatment gut microbiota, the transplanted gut communities were highly similar among recipients post-transplantation, were indistinguishable from that of the donor, and were rich in members of Blautia, Coprococcus, and Faecalibacterium. In each case, the gut microbiota restoration led to a complete patient recovery and symptom alleviation.

Conclusion: We conclude that $C$. difficile infection can be successfully treated by fecal microbiota transplantation and that this leads to stable transformation of the distal gut microbial community from the one abundant in aerotolerant species to that dominated by members of the Clostridia.

Keywords: Microbiota, Microflora, Clostridium difficile, Fecal microbiota transplantation, Microbiota Array

\section{Background}

The use of antibiotics in modern medicine has led to a significant inhibition and in some cases complete eradication of many infectious agents that threaten human population [1]. However, the recent spread of broad-spectrum

\footnotetext{
*Correspondence: oleg.paliy@wright.edu; sadowsky@umn.edu

${ }^{\dagger}$ Equal contributors

'Department of Biochemistry and Molecular Biology, Boonshoft School of Medicine, Wright State University, 3640 Col. Glenn Hwy, Dayton, OH 45435, USA ${ }^{2}$ Department of Soil, Water, and Climate, Biotechnology Institute, and Microbial Plant and Genomics Institute, University of Minnesota, 140 Gortner Lab, 1479 Gortner Avenue, St. Paul, MN 55108, USA

Full list of author information is available at the end of the article
}

antibiotic use is also linked to an increase in the incidence of antibiotic-associated intestinal disease. Many of these incidents are caused by Clostridium difficile, an opportunistic human intestinal pathogen from class Clostridia. Clostridium difficile infection (CDI) is known to have a range of manifestations, from mild diarrhea to fulminant colitis, toxic megacolon, and death. The disease often manifests itself after treatment with antibiotics and the associated loss of resident microbiota in the intestine. Resistance of $C$. difficile to many classes of antibiotics and its ability to form spores allows this bacterium to survive antibiotic administration 
better than many commensal species. The decrease in commensals in the gut creates conditions favorable for a subsequent overgrowth of this opportunistic pathogen [2]. C. difficile spores are often acquired nosocomially, and as a result, a high incidence of CDI is seen among hospitalized patients, in the outpatient community, and among nursing home residents $[3,4]$. Other reservoirs of CDI can also exist according to a recent report [5].

Because of the resistance of $C$. difficile spores to antibiotics, it is challenging to cure CDI with antibiotic administration. Among alternative strategies, fecal microbiota transplantation (FMT) is gaining a wider acceptance as treatment for recurrent CDI. In this technique, fecal microbiota obtained from a healthy donor is processed, standardized, and subsequently transplanted into patients suffering from the recurrent $C$. difficile infection. FMT is highly successful (>90\% success rate) and CDI symptoms often resolve within days of the transplantation procedure $[6,7]$. Recent studies from our group showed that the eradication of the disease symptoms is accompanied by a dramatic shift in the microbial community as examined by TRFLP and gene sequencing analyses $[8,9]$. However, these methods were limited in the achieved taxonomic resolution and the ability to directly quantify microbiota members, and thus they could not reveal detailed microbiota composition before and after FMT treatment.

In this study we used a human intestinal microbiotaspecific phylogenetic Microbiota Array [10-13] to measure phylotype- and genus-level changes in gut microbiota of three CDI patients who underwent an FMT procedure. Microbiota Arrays contain probes targeting full-length 16S rRNA genes of 775 human microbiota phylotypes and allows direct comparison of taxon abundances between samples [10]. The microarray data were corroborated with Illumina high-throughput sequencing and fluorescent in situ hybridization.

\section{Methods \\ Patients}

All patients suffered from multiple recurrent $C$. difficile infection (CDI) refractory to clearance by standard antibiotic therapies, as defined previously [14]. The study of their fecal microbiota before and after FMT was approved by the University of Minnesota Institutional Review Board and all patients provided informed consent to participate in this study.

\section{FMT procedure}

The FMT was performed using a standardized preparation of concentrated fecal microbiota as previously described [14]. Criteria for the selection of donor were described in detail previously $[9,14]$. The same donor was used for all recipients, but individual donations were collected on different days. Briefly, $50 \mathrm{~g}$ of fecal material were mixed with
$250 \mathrm{~mL}$ of sterile phosphate buffered saline (PBS). The feces were blended, sieved, and the resulting suspension was centrifuged and washed in PBS. Patient 1 received freshly prepared material, while frozen lots were used for patients 2 and 3 . We previously demonstrated FMT success with frozen fecal microbial suspension to be comparable to that obtained with a fresh preparation [14]. The patients were treated with $125 \mathrm{mg}$ vancomycin, four times daily by mouth, until 2 days prior to the procedure. The day before the procedure, patients received a split dosage polyethylene glycol-based purgative $\left(\right.$ GoLYTELY $\left.^{\oplus}\right)$ to remove residual antibiotic and fecal material. FMT was performed via colonoscopy as previously described [14].

\section{Sample collection}

Patient fecal samples were collected at home by the patients and stored frozen at approximately $-20^{\circ} \mathrm{C}$. The first sample for each patient was collected while the patient was receiving oral vancomycin $(125 \mathrm{mg}$, four times per day) during the period up to the FMT. Samples were transferred to the laboratory within 1 week of collection and stored at $-80^{\circ} \mathrm{C}$ until used. Donor samples for DNA extraction were collected during processing of material for FMT, and stored frozen at $-80^{\circ} \mathrm{C}$ until used. A timeline showing sample collection for the three patients involved in this study is shown in Figure 1A.

\section{Isolation of genomic DNA and hybridization to Microbiota Array}

Total genomic DNA (gDNA) was isolated from fecal material using ZR Fecal DNA Isolation kit (Zymo Research Corporation, procedure incorporates bead beating) according to manufacturer's protocol. The full length bacterial 16S rRNA gene was amplified from genomic DNA with degenerate primers Bact-27Fv4 (5'-AGRGTTYGA TYMTGGCTCAG-3') and Univ-1492Rv1 (5'-GGHTA CCTTGTTACGACTT-3') [10,15]. Four separate PCR reactions were pooled together, fragmented, and then hybridized to Microbiota Array. Microbiota Array is based on Affymetrix, Inc., platform and contains sets of phylogenetic $16 \mathrm{~S}$ rRNA gene probes (25-mer probes, 5 to 11 probe pairs in each set, each probe pair consists of perfect match and mismatch probes, each set interrogates a separate phylotype) allowing detection and enumeration of 775 bacterial phylotypes of human intestinal microbiota [10]. Microarray hybridization, washing, and scanning were carried out as described previously $[12,16]$.

\section{Microarray data analysis}

Raw microarray data were analyzed using previously developed pipeline [12]. Specifically, to obtain phylotype detection calls, the raw data were processed with standard MAS5 detection algorithm (Affymetrix, Inc.) using $\alpha 1=0.03$ and $\alpha 2=0.05$ parameter values. MAS5 algorithm is based on 


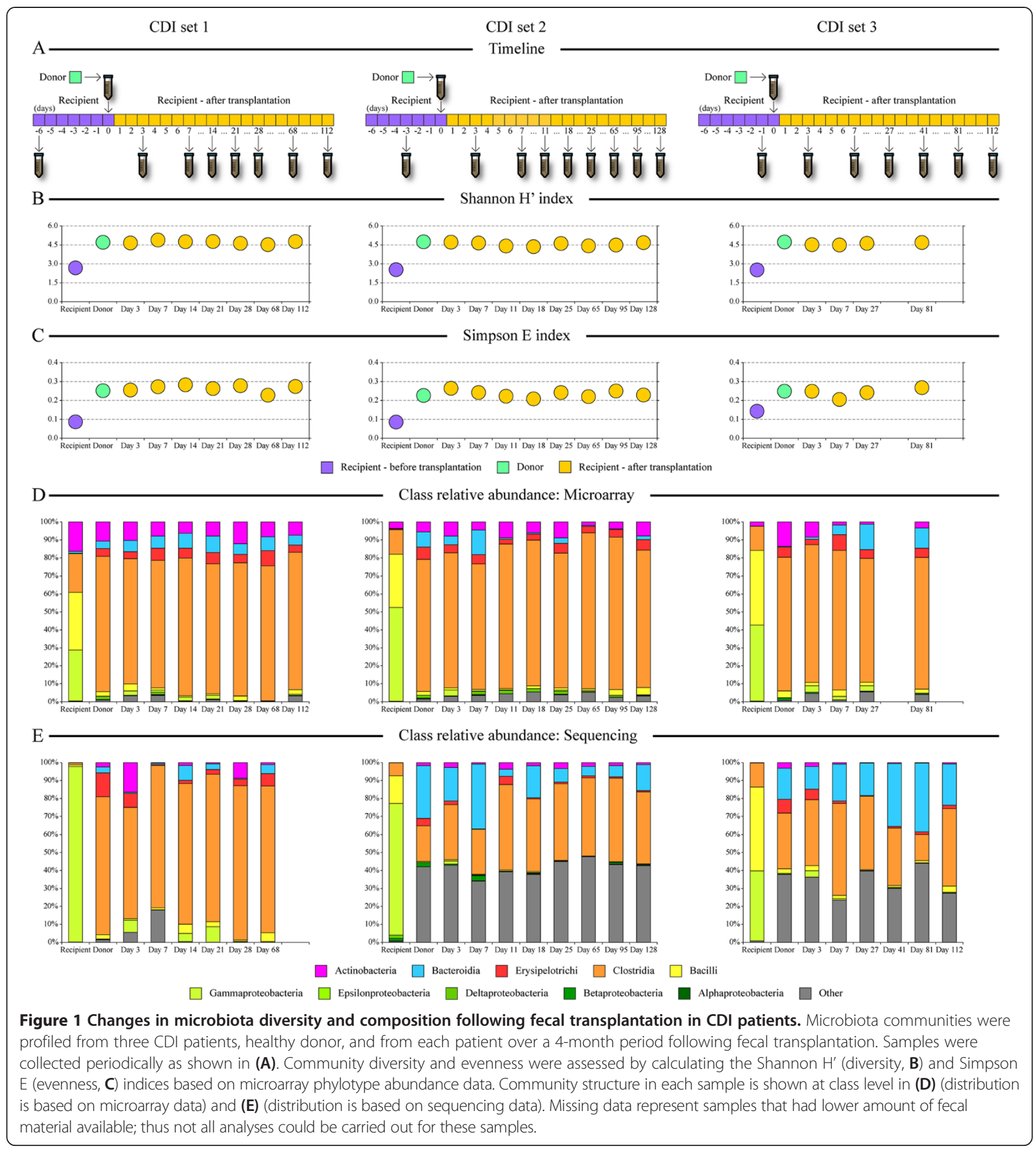

the Wilcoxon's rank test. To obtain hybridization signal estimates for each phylotype, raw data were first normalized in CARMAweb utilizing MAS5-VSN-MAS5-MedianPolish procedure as we did previously [10]. The normalized phylotype signal values were adjusted for estimated cross-hybridization rate and unequal 16S rRNA gene copy number as described [12]. To assess our ability to separate samples based on their microbial phylotype composition, principal components (PCA) and phylogenetic principal coordinates ( $\mathrm{PCoA})$ analyses were used [13]. PCA, alpha diversity calculations, and permutation analyses were performed in Matlab (The Mathworks, Inc.) by employing custom written scripts. PCoA analyses were carried out on the Fast UniFrac web server [17]. Separation of genera into clusters according to genus abundances among samples was based 
on the K-means clustering method with manual curation to separate genera displaying patient-specific patterns of abundance changes.

\section{High-throughput amplicon sequencing and data analysis} DNA extractions were done as previously described [9] using MOBIO PowerSoil DNA extraction kits (MOBIO, Carlsbad, CA, USA), according to the manufacturer's instructions. Fecal DNA samples were used as template in PCR amplification reactions of the $\mathrm{V} 6$ hypervariable region of the 16S rRNA gene. All PCR reactions used $25 \mathrm{ng}$ of fecal DNA as template and were performed in triplicates.

DNA and amplicon preparation for high-throughput sequencing were carried out as described [9]. The samples were sequenced using Illumina Hiseq 2000 sequencer following the manufacturer's protocols (Illumina, Hayward, CA, USA). Paired-end sequences were generated (100 nt read length) with one to three pooled samples per lane following Illumina multiplexing protocols. Paired ends were merged using a custom $\mathrm{C}$ script [9] by employing a minimal overlap of 25 nucleotides with $98 \%$ identity. Sequence data were processed and analyzed using the MOTHUR program [18]. Merged sequences were binned into individual sample sets according to the six nucleotide barcode sequences. The list of barcodes used and primers is provided in Additional file 1: Table S1. Sequence reads containing ambiguous bases, homopolymers larger than seven nucleotides, more than one mismatch in the primer sequence, or an average per base quality score below 25 were removed. Primer and barcode sequences were trimmed from the sequence reads prior to analysis. Sequences that only appeared once in the total set were assumed to be a result of sequencing error and were removed from the analysis. Sequences that were flagged as likely chimeras using the UCHIME algorithm were also removed from the analysis. Sequences were clustered into OTUs using the furthest neighbor algorithm with a $90 \%$ cutoff [19]. Taxonomic assignment was done using the Bayesian method with a 100 iteration bootstrap algorithm and a probability cutoff of 0.60 [9]. Summary of the obtained reads and OTU assignments are provided in the Additional file 2: Table S2. The complete sequence dataset is available in the SRA archive under bioproject number PRJNA238486 and metadata are presented in Table S6.

\section{Fluorescent in situ hybridization (FISH)}

FISH was carried out as we did previously [11]. Clostridia, Bacteroidia, and Proteobacteria were visualized using FITC-labeled probes Clept1240 (5'-GTTTTRTCAACGG CAGTC-3') + Erec482 (5' -GCTTCTTAGTCARGTACC G-3'), Bac303 (5'-CCAATGTGGGGGACCTT-3'), and Prot612 (5'-TTCCCVGGTTRAGCCCKGG-3'), respectively [11]. Following a previously optimized sample preparation protocol [11], cells were visualized under Nikon TE2000-S fluorescent microscope. In order to conduct valid comparisons between FISH and microarray results, microarray data were adjusted to account for the inability of FISH probes to detect certain genera of Clostridia and Bacteroidia (see [11] for details).

\section{Results}

\section{Outcomes of the FMT procedure}

We profiled intestinal microbiota in fecal samples collected from (1) three CDI patients before the FMT procedure, from (2) healthy donor, and from (3) each CDI patient over a period of 4 months following the FMT procedure. Samples were collected from each CDI patient a few days before the transplantation, on days 3 and 7 after transplantation, and then periodically with gradually increasing time periods between sample collections based on patients' availability and ability to provide a fresh stool sample (Figure 1A). Clinically, all three CDI patients had reduced diarrheal symptoms within several days after the FMT. All patients were documented to have undetectable $C$. difficile toxin B at 2 months by qPCR. Patients 1 and 3 had a firm bowel movement on day 3 and remained free of $C$. difficile infection for 2.5 and 2.0 years, respectively. Patient 2 had underlying ulcerative colitis, which decreased from severe pancolitis to moderate disease as evidenced by endoscopic and histological criteria 1 month after FMT. Clinically, bowel movement frequency decreased from 10-12/day to $4 /$ day, and rectal bleeding and tenesmus resolved. Colonoscopy at 1 year showed endoscopically moderate disease in the distal colon, but near normal appearance in the proximal colon. This patient remained free of C. difficile infection for 1.5 years until treated with antibiotics for a urinary tract infection, at which time he experienced a re-infection with $C$. difficile.

\section{Changes in distal gut microbial community composition in CDI patients following fecal transplantation}

The microarray phylotype abundance data were used to assess the differences in the intestinal microbial communities among all samples. The ecological organization of the communities was calculated using the Shannon's diversity (Figure 1B) and the Simpson's evenness (Figure 1C) indices. Overall, the gut microbiota in CDI patients had low diversity and evenness in all three patients prior to FMT (Shannon's H' $=2.58 \pm 0.09$ and Simpson $E=0.11 \pm 0.03$ ). The communities were dominated by relatively few members with high abundance, and the overall number of species in the community was low. In contrast, the microbiota from the healthy donor showed statistically significantly higher diversity and evenness $\left(H^{\prime}=4.73 \pm 0.02\right.$ and $E=$ $0.24 \pm 0.01 ; \alpha<10^{-5}$ and $\alpha=0.001$, respectively, based on a one-tail $T$-test). After-transplantation samples from day 3 
onward had increased diversity and evenness similar to those of the donor microbiota $\left(\mathrm{H}^{\prime}=4.61 \pm 0.14\right.$ and $\mathrm{E}=$ $0.25 \pm 0.02 ; \alpha=0.18$ and $\alpha=0.74$ based on a two-tail $T$-test of donor and after-FMT sample comparison). Similar effects of microbiota transplantation (delivered as duodenal infusion) on gut microbial diversity in CDI patients were also recently observed by van Nood and co-workers [7]. The overall community structure remained remarkably stable through the 4-month sampling period (average Spearman correlation of microarray-determined genus abundances between consecutive time points was 0.87 for after transplantation samples) and at the end of the observation both community diversity and evenness remained similar to that of the donor $\left(\mathrm{H}^{\prime}=4.71 \pm 0.05\right.$ and $\mathrm{E}=0.26 \pm 0.02$ ).

At the class level, microarray results revealed considerable changes between the microbiota profiles before and after transplantation (Figure 1D and Additional file 3: Table S3). In all three CDI patients, their fecal samples before transplantation contained relatively high abundance of organisms belonging to classes Gammaproteobacteria (40.9\% cumulative abundance on average) and Bacilli (34.5\% on average). Donor samples were dominated by the classes Clostridia (74.5\%), Actinobacteria (10.0\%), Erysipelotrichi (5.6\%), and Bacteroidia (4.3\%). Concordantly, the recipients' after-transplantation samples showed increases in Bacteroidia (from 0.5\% to 6.1\% on average) and Clostridia (from $16.1 \%$ to $75.5 \%$ ), with Proteobacteria $(2.1 \%)$ and Bacilli $(2.0 \%)$ in low abundance. The microarray class level data also indicated an overall stability of the microbial community structure in the after-transplantation distal gut over the entire follow-up period (Figure 1D). The dramatic shifts in the abundances of microbial classes following FMT were also evidenced by Illumina sequencing (Figure 1E), although the sequencing results showed somewhat greater community variability among the after-transplantation samples. The observed greater robustness of microarray data is likely explained by the ability of microarray to measure presence and abundance of each interrogated phylotype in each sample [12]. Thus, the microarray results are not dependent on the sequencing depth and are less sensitive to any $16 \mathrm{~S}$ rRNA gene PCR amplification biases.

Utilizing phylotype abundance data obtained with Microbiota Array, sampled microbial communities were also analyzed with high-dimensionality reducing principal components analysis (PCA) and phylogenetic principal coordinates analysis (PCoA) ordination approaches [13]. All ordination analyses showed a clear separation of recipients' before-transplantation samples from those of the donor and the patients' after-transplantation samples (Figure 2). Consistent with our analysis as described above, there was a considerable difference in the community phylotype structure between the CDI and donor samples, and this difference was the largest determinant of dataset variability, because the donor and CDI samples were separated along the principal component/coordinate 1 axis representing the highest data variability [20]. A significant degree of variability was seen among the recipients before transplantation especially in the phylotype presence (Figure 2B), indicating that pre-transplantation gut microbial communities were unique to each profiled CDI patient. The after-transplantation samples for all three recipients clustered together with donor samples, demonstrating that compositional individuality of pre-treatment gut microbiota had little influence on the post-FMT
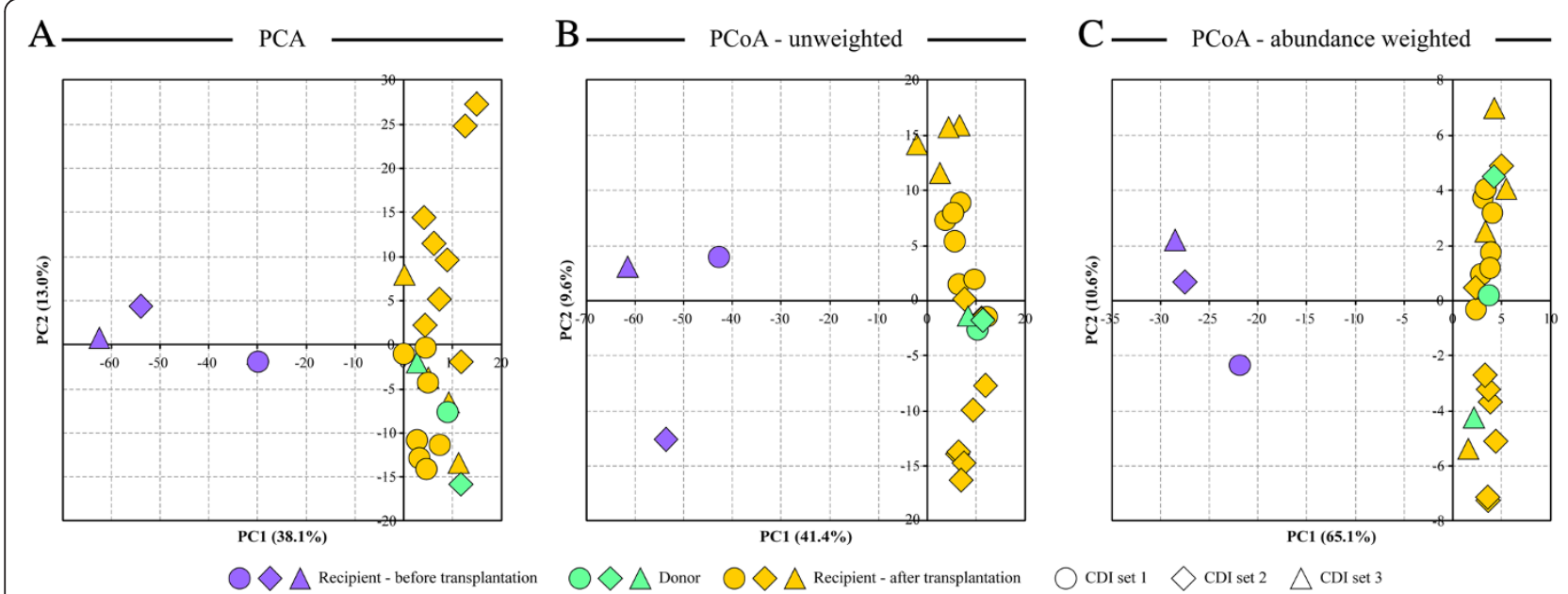

Figure 2 Separation of samples based on ordination multivariate analysis of microarray phylotype abundance data. Principal components analysis (PCA, A) and unweighted (separation is based on phylotype presence, B) and weighted (separation is based on phylotype presence and abundance, $\mathbf{C}$ ) principal coordinates analysis (PCOA) show separation of recipient samples before transplantation from both donor and recipient samples obtained after transplantation. Percent of dataset variability explained by each principal component/coordinate is shown in brackets in axis titles. 
microbial community structure. Post-FMT distal gut microbiota structure was thus determined by donor microbiota community.

\section{Visualization of fecal microbiota with FISH}

Because fluorescent in situ hybridization allows cell quantitation through direct visualization, it is a good choice for validation of results obtained through DNAbased techniques such as phylogenetic microarrays or high-throughput sequencing. With that goal, we utilized FISH to visualize and quantify Bacteroidia, Clostridia, and Proteobacteria in three fecal samples from CDI set 1: recipients before transplantation, donor, and samples obtained from the same patient 3 days after transplantation. As shown in Figure 3A, the taxon abundances obtained from FISH quantitation matched well those from the microarray results, with only somewhat higher estimate of Proteobacteria in the recipient pre-transplantation sample based on FISH (28.8\% and $41.0 \%$ based on array and FISH calculations, respectively, note that microarray data were adjusted to match each FISH probe inability to detect some members of each class/phylum). A representative image from each sample examined by FISH using a Proteobacteria probe and a generic DNA stain (DAPI) are shown in Figure 3B; Proteobacteria cells were largely detected only on a slide with initial recipient microbiota but not in the other two samples.

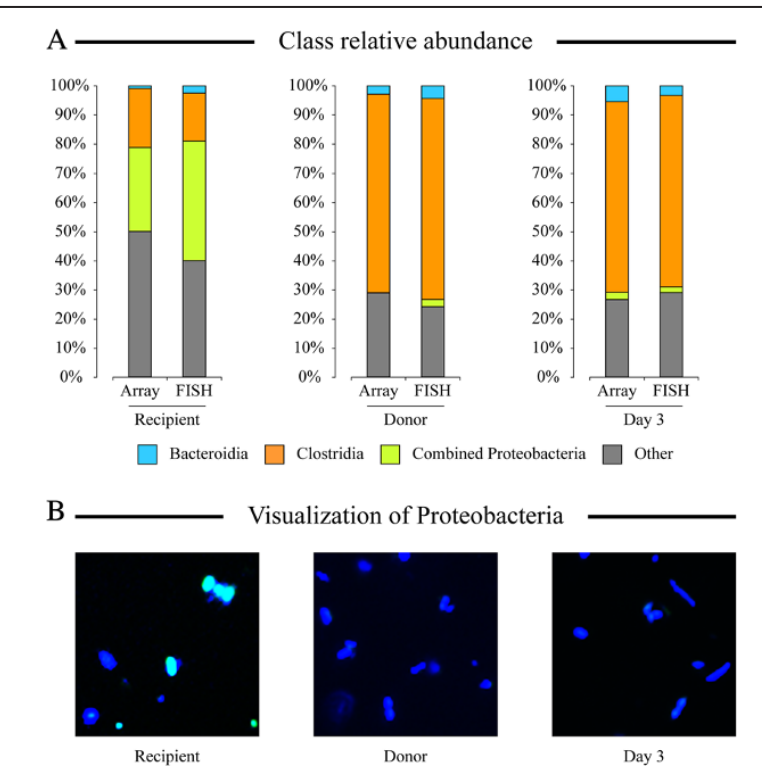

Figure 3 Quantification of bacterial abundances using

fluorescent in situ hybridization. Select fecal samples from CDI set 1 were profiled using class specific DNA probes as shown. (A) A comparison of class relative abundances measured by Microbiota Array and by FISH. (B) Representative captured image from each sample visualized with Proteobacteria fluorescent probe (green color) and DAPI DNA stain (blue color).

\section{Specific genera are responsible for observed microbiota differences among samples}

Because Microbiota Arrays contain probes to individual microbial phylotypes, its use allowed a quantitative assessment of phylotype and genus level abundances in all samples. We thus sought to compare genus abundances among samples and to define groups of genera that displayed similar patterns across the sample set. Genera were distributed into five groups based on their abundance values among samples (Figure 4, Additional file 4: Figure S1, and Additional file 5: Table S4). Group 1 comprised genera that were present at high abundance in the donor and aftertransplantation samples but which were not highly abundant in CDI patients' samples prior to FMT procedure. Notable members included a number of genera from the classes Clostridia and Bacteroidia, such as Bacteroides, Blautia, Coprococcus, Faecalibacterium, Papillibacter, and Roseburia. These taxa are known to comprise a substantial portion of human colonic microbiota and to play important roles in the energy metabolism and commensal host-microbial interactions [21]. Among these, only Blautia and Coprococcus were also somewhat abundant in the patient beforetransplantation samples (Figure 4, group 1).

Group 2 consisted of five facultatively anaerobic and/or aerotolerant genera Enterobacter, Escherichia, Lactobacillus, Raoultella, and Veillonella; these were present at high abundance in all three before-transplantation samples, but not in donor samples. The levels of these genera were similarly low in the fecal samples of CDI patients after FMT. Genera that we assigned to group 3 were abundant in some but not all before-transplantation samples, while their levels in donor and after-transplantation samples were similarly low. These included Streptococcus (abundant in patients 1 and 2), Zymophilus and Klebsiella (abundant in patients 2 and 3), Haemophilus (only found in patient 1), and Enterococcus (only in patient 3). Two genera - Bifidobacterium and Lactococcus - did not show consistent patterns across donor, before-, and after-transplantation samples and were thus assigned to group 4.

Group 5 comprised genera that were not consistently detected in samples or detected with very low abundance ( $N=86$, see Additional file 4: Figure S1). Similar to the findings shown in Figure 1, genera present in high abundance in the donor samples were also highly abundant in the after-transplantation samples. Conversely, genera present in high abundance in all or some of the recipient before-transplantation samples were in relatively lower abundance in both the donor and after-transplantation samples. This indicates a complete reorganization of the gut microbiota in CDI patients following FMT procedure.

\section{Species level changes following FMT}

The distribution of phylotypes and species generally followed the pattern observed for genera, with species of 


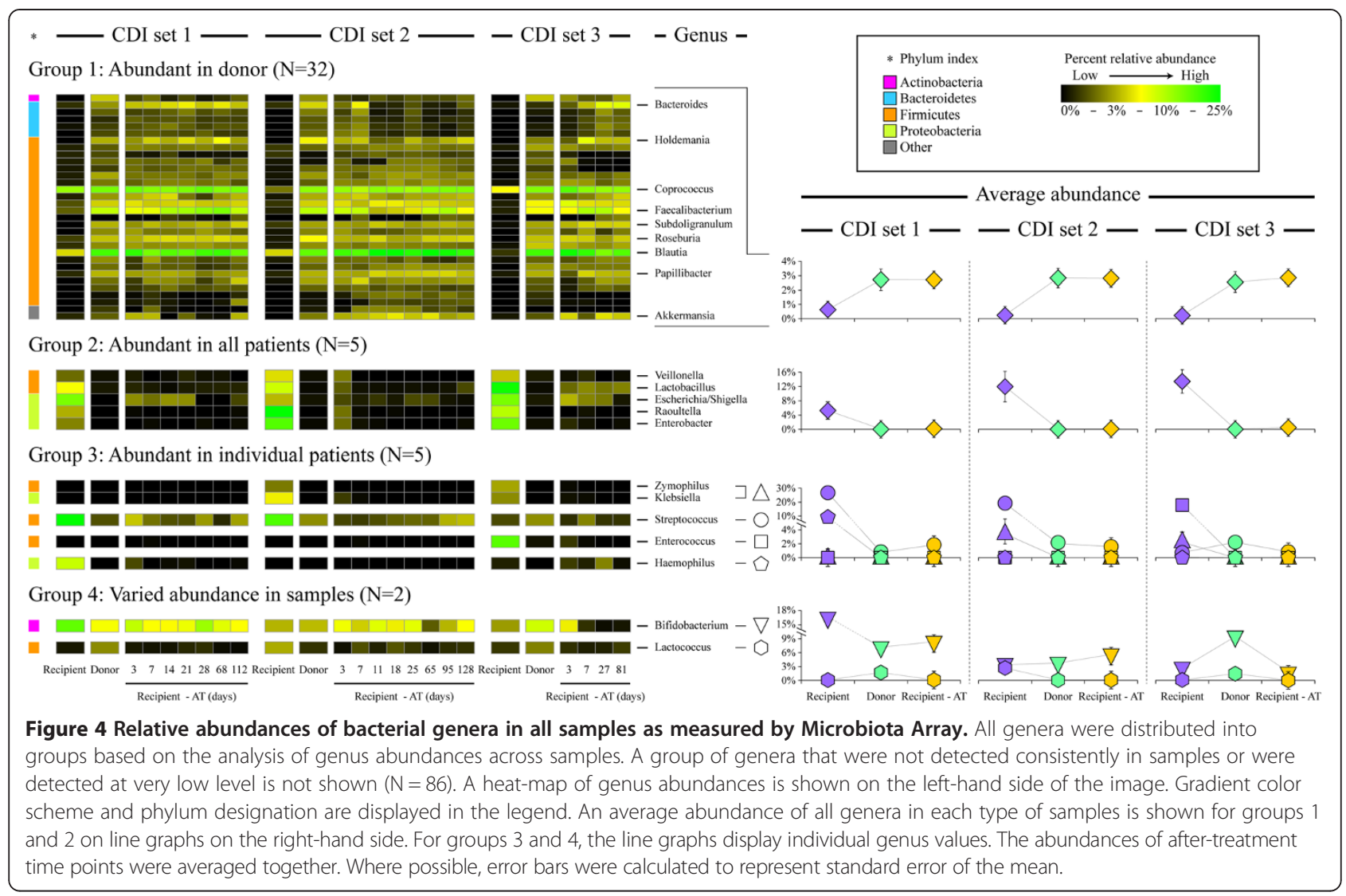

Clostridia and Bacteroidia generally scarce or undetected in pre-FMT samples but abundant in donor and afterFMT samples. While microarray allows enumeration of 775 different microbial phylotypes, only about 65 of them are assigned to known microbial species [10]. Among these, Bacteroides fragilis, B. ovatus, and B. uniformis were not present above $0.1 \%$ abundance level in any of the original patient samples, but increased to an average cumulative abundance of $1.9 \%$ after transplantation. Similar increases in after-FMT samples were observed for Faecalibacterium prausnitzii, Clostridium bartlettii, Dorea longicatena, Holdemania filiformis, Roseburia intestinalis, and Ruminococcus obeum. Interestingly, even though the microarrays contained a probe-set for Clostridium difficile, this species was not confidently detected in any of the original CDI patients' fecal samples, possibly because the vegetative $C$. difficile cells were largely eradicated by antibiotic treatment, whereas DNA isolation from $C$. difficile spores might not have been successful [22]. Concordantly, C. difficile toxin $\mathrm{B}$ was also not detected in these samples with clinical qPCR test. In contrast, individual patient's pretransplantation samples contained remarkably high abundance levels of a few other species: - Bifidobacterium adolescentis (12.8\% of overall abundance) and Escherichia coli $(3.5 \%)$ in patient 1, Klebsiella pneumonia (5.5\%) and
Bifidobacterium adolescentis (3.1\%) in patient 2, and Enterococcus faecium (17.1\%), Lactobacillus salivarius (6.7\%), and Escherichia coli (3.3\%) in patient 3.

\section{Discussion}

The goal of this study was to profile phylotype-level microbiota composition in three CDI patients and to follow microbiota changes during and after FMT. While several previous reports have evaluated microbiota alterations following FMT therapy in human patients [7-9,23,24], the genus and species level changes were explored only in few studies [25]. The utilization of phylogenetic Microbiota Array allowed us to obtain quantitative measurements of different taxonomical groups in all samples, and microarrays uniquely generated genus and species level resolution of distal gut microbiota in these samples. We used Illumina high-throughput sequencing and FISH to provide additional support for our findings.

The analyses presented here show that the colon of each CDI patient was host to a severely compromised intestinal microbial community, which was significantly reduced in diversity and richness. This was likely a result of $C$. difficile proliferation as well as due to the antibiotic treatment used in an attempt to rid the disease. Ulcerative colitis, a common coexisting condition in patients with recurrent CDI $[14,26]$, may have contributed to dysbiosis in patient 
2 , although typically ulcerative colitis alone does not significantly decrease microbial diversity [27]. While the healthy gut microbiota is usually dominated by Clostridia and Bacteroidia, fecal samples of the CDI patients were abundant in Gammaproteobacteria and Bacilli. Interestingly, both of these abundant taxons contain many facultative anaerobic or aerotolerant bacteria, and many are known to be abundant in the human ileum, in part due to their ability to tolerate the presence of oxygen [28-33]. Normally such species contain genes coding for proteins that allow cells to ameliorate toxic reactive oxygen species (ROS), such as hydrogen peroxide and superoxide $\mathrm{O}_{2}^{-}$. In addition to the tolerance against molecular oxygen and its effects, these oxidative stress response genes also provide protection against ROS generated by neutrophils and macrophages and released into the gut during inflammation [34]. We found the presence of at least two of four genes coding for either catalase (decomposes hydrogen peroxide), superoxide dismutase (detoxifies superoxide), glutathione synthase (glutathione serves as antioxidant), or glutathione peroxidase (reduces hydrogen peroxide to water) in the genome sequences of human gut representatives of Enterobacter, Escherichia, Lactobacillus, Veillonella, Klebsiella, Haemophilus, Enterococcus, and Streptococcus (see Additional file 6: Table S5). These genera were found to be highly abundant in the fecal samples of CDI patients, an observation partially matched by the analysis of CDI microbiota by Anthraham and colleagues [35]. In contrast, the genomes of several prominent obligate anaerobes from the human gut such as Faecalibacterium prausnitzii and Roseburia intestinalis did not contain any of these genes.

We offer three potential explanations for the observed composition of the fecal microbiota in CDI patients. 1) Because $C$. difficile infection is associated with frequent diarrhea, it usually leads to a significantly shortened transit time in the large intestine [36], and diarrhea would result in a lower overall load of microbes in the colon [37]. This may cause significantly fewer members of the colonic communities being present in the fecal material, and as a consequence it will lead to an increase of relative abundances of small intestinal genera in feces (these can be shed in higher amounts into the colonic lumen due to faster gut transit time). 2) Because diarrhea in combination with antibiotic administration during standard CDI treatment are expected to reduce the overall counts of microbes in the colon [38], this may increase the average oxygen level in that gut region (typically less than 1\% [39]). Additionally, reactive oxygen species can be released in the gut of CDI patients by macrophages and neutrophils, which are recruited into gut mucosa during CDI development [40]. Both of the described effects will create an environment selectively more advantageous for the species that are able to tolerate the presence of oxygen and ROS. 3) Antibiotic administration alone can play a role in shaping the composition of the intestinal microbial community. A standard antibiotic regimen in CDI included metronidazole followed by multiple cycles of vancomycin, and patient 2 also received rifaximin. Since metronidazole predominantly targets anaerobic bacteria [41], and vancomycin inhibits cell wall synthesis of gram-positive bacteria [42], the use of these antibiotics would be expected to reduce members of class Clostridia (most are strictly anaerobic gram-positive species) and not affect Proteobacteria (many are facultatively anaerobic gram-negative species). It is likely that a combination of the factors described impart a selective pressure on the gut microbial communities in CDI patients. This in turn likely leads to a relative decrease in the abundance of obligate anaerobic species and an increase in the presence of aerotolerant members in the distal gut and fecal matter.

In contrast to the fecal communities of CDI patients, the fecal samples of the donor were dominated by members of Clostridia, Actinobacteria, Erysipelotrichi, and Bacteroidia, which is a common composition of the distal gut microbiota in healthy humans [43]. The FMT procedure rapidly and drastically altered the intestinal microbiota communities in CDI patients, both in the taxonomy of organisms present and in their relative abundances, so that even at day 3 after FMT the recipient's microbiota matched that of the donor. Such microbiota restructuring after FMT was also noted in our previous reports as well as in several other studies $[8,9,24,44]$. Genus and species level changes in CDI patients upon FMT were also reported by Shahinas and colleagues, though the success of community reorganization varied from case to case in that study [25]. In all our patients, the composition of these engrafted communities remained stable over the 4-month observation period, and the structure of the microbiota depended upon the original composition of the donor community. This microbial compositional shift was accompanied by the cessation of CDI symptoms.

\section{Conclusion}

Several reports have indicated the overwhelming clinical efficacy of FMT $[7,14,45,46]$. In contrast to probiotic therapy, which introduces a limited number of microorganisms into the intestinal tract, FMT effectively replaces the entire colonic microbiota with a healthy one in order to reestablish the lost intestinal homeostasis. The success of FMT to treat $C$. difficile infection, revealed in this and other recent studies, also opens possibilities for application of this approach to other gastrointestinal disorders. Conditions such as inflammatory bowel disease and metabolic disorders including malnutrition and obesity, where the resident gut microbiota is thought to be a key contributing 
factor, are possible candidates to which FMT could be applied with success $[47,48]$.

\section{Additional files}

Additional file 1: Table S1. Primer sequences and barcodes used for Illumina-based sequencing.

Additional file 2: Table S2. Cumulative number of Illumina reads and assigned OTUs per sample.

Additional file 3: Table S3. Class level abundances among all profiled samples.

Additional file 4: Figure S1. Expanded version of Figure 4 showing all 130 profiled microbial genera.

Additional file 5: Table S4. Genus level abundances among all profiled samples.

Additional file 6: Table S5. Presence of ROS detoxifying genes among select genera of human gut microbiota.

\section{Abbreviations}

CDI: C. difficile infection; FISH: Fluorescent in situ hybridization; FMT: Fecal microbiota transplantation; gDNA: Genomic DNA; PCA: Principal components analysis; PCoA: Principal coordinates analysis; ROS: Reactive oxygen species.

\section{Competing interests}

The authors declare that they have no competing interests.

\section{Authors' contributions}

AIK, OP, and MJS have developed study concept and design; VS, MJH, AmK, and TU acquired the data; VS, MJH, and OP analyzed the data; VS, MJH, MJS, and OP wrote the manuscript. All authors read and approved the final manuscript.

\section{Acknowledgements}

The work described in this manuscript was supported by the National Institutes of Health grants AT003423 to OP and Al091907 to AIK and MJS.

\section{Author details}

${ }^{1}$ Department of Biochemistry and Molecular Biology, Boonshoft School of Medicine, Wright State University, 3640 Col. Glenn Hwy, Dayton, OH 45435, USA. ${ }^{2}$ Department of Soil, Water, and Climate, Biotechnology Institute, and Microbial Plant and Genomics Institute, University of Minnesota, 140 Gortner Lab, 1479 Gortner Avenue, St. Paul, MN 55108, USA. 'Division of Gastroenterology; Department of Medicine, Center for Immunology, University of Minnesota, Minneapolis, MN, USA.

Received: 10 November 2013 Accepted: 12 March 2014 Published: 21 April 2014

\section{References}

1. Khardori N: Antibiotics-past, present, and future. Med Clin North Am 2006, 90:1049-1076.

2. Kelly CP, LaMont JT: Clostridium difficile infection. Annu Rev Med 1998, 49:375-390.

3. Arvand M, Moser V, Schwehn C, Bettge-Weller G, Hensgens MP, Kuijper EJ: High prevalence of Clostridium difficile colonization among nursing home residents in Hesse, Germany. PLOS ONE 2012, 7:e30183.

4. Curry SR, Muto CA, Schlackman JL, Pasculle AW, Shutt KA, Marsh JW, Harrison LH: Use of multilocus variable number of tandem repeats analysis genotyping to determine the role of asymptomatic carriers in Clostridium difficile transmission. Clin Infect Dis 2013, 57:1094-1102.

5. Eyre DW, Cule ML, Wilson DJ, Griffiths D, Vaughan A, O'Connor L, Ip CL, Golubchik T, Batty EM, Finney JM, Wyllie DH, Didelot X, Piazza P, Bowden R, Dingle KE, Harding RM, Crook DW, Wilcox MH, Peto TEA, Walker AS: Diverse sources of $C$. difficile infection identified on whole-genome sequencing. N Engl J Med 2013, 369:1195-1205.

6. Bakken JS, Borody T, Brandt L, Brill JV, Demarco DC, Franzos MA, Kelly C, Khoruts A, Louie T, Martinelli LP, Moore TA, Russell G, Surawicz C, Fecal Microbiota Transplantation Group: Treating Clostridium difficile infection with fecal microbiota transplantation. Clin Gastroenterol Hepatol 2011, 9:1044-1049.

7. van Nood E, Vrieze A, Nieuwdorp M, Fuentes S, Zoetendal EG, de Vos WM, Visser CE, Kuijper EJ, Bartelsman JF, Tijssen JG, Speelman P, Dijkgraaf MG, Keller JJ: Duodenal infusion of donor feces for recurrent Clostridium difficile. N Engl J Med 2013, 368:407-415.

8. Khoruts A, Dicksved J, Jansson JK, Sadowsky MJ: Changes in the composition of the human fecal microbiome after bacteriotherapy for recurrent Clostridium difficile-associated diarrhea. J Clin Gastroenterol 2010, 44:354-360.

9. Hamilton MJ, Weingarden AR, Unno T, Khoruts A, Sadowsky MJ: Highthroughput DNA sequence analysis reveals stable engraftment of gut microbiota following transplantation of previously frozen fecal bacteria. Gut Microbes 2013, 4:125-135.

10. Paliy $\mathrm{O}$, Kenche $\mathrm{H}$, Abernathy F, Michail S: High-throughput quantitative analysis of the human intestinal microbiota with a phylogenetic microarray. App/ Environ Microbiol 2009, 75:3572-3579.

11. Rigsbee L, Agans R, Shankar V, Kenche H, Khamis HJ, Michail S, Paliy O: Quantitative profiling of gut microbiota of children with diarrhea-predominant Irritable Bowel Syndrome. Am J Gastroenterol 2012, 107:1740-1751.

12. Rigsbee $L$, Agans $R$, Foy BD, Paliy $O$ : Optimizing the analysis of human intestinal microbiota with phylogenetic microarray. FEMS Microbiol Ecol 2011, 75:332-342.

13. Shankar V, Agans R, Holmes B, Raymer M, Paliy O: Do gut microbial communities differ in pediatric IBS and health? Gut Microbes 2013, 4:347-352.

14. Hamilton MJ, Weingarden AR, Sadowsky MJ, Khoruts A: Standardized frozen preparation for transplantation of fecal microbiota for recurrent clostridium difficile infection. Am J Gastroenterol 2012, 107:761-767.

15. Paliy O, Foy B: Mathematical modeling of $16 \mathrm{~S}$ ribosomal DNA amplification reveals optimal conditions for the interrogation of complex microbial communities with phylogenetic microarrays. Bioinformatics 2011, 27:2134-2140.

16. Agans R, Rigsbee L, Kenche H, Michail S, Khamis HJ, Paliy O: Distal gut microbiota of adolescent children is different from that of adults. FEMS Microbiol Ecol 2011, 77:404-412.

17. Hamady M, Lozupone C, Knight R: Fast UniFrac: facilitating highthroughput phylogenetic analyses of microbial communities including analysis of pyrosequencing and PhyloChip data. ISME J 2010, 4:17-27.

18. Schloss PD, Westcott SL, Ryabin T, Hall JR, Hartmann M, Hollister EB, Lesniewski RA, Oakley BB, Parks DH, Robinson CJ, Sahl JW, Stres B, Thallinger GG, Van Horn DJ, Weber CF: Introducing mothur: open-source, platformindependent, community-supported software for describing and comparing microbial communities. Appl Environ Microbiol 2009, 75:7537-7541.

19. Cole JR, Wang Q, Cardenas E, Fish J, Chai B, Farris RJ, Kulam-Syed-Mohideen AS, McGarrell DM, Marsh T, Garrity GM, Tiedje JM: The ribosomal database project: improved alignments and new tools for rRNA analysis. Nucleic Acids Res 2009, 37:D141-145.

20. Ramette A: Multivariate analyses in microbial ecology. FEMS Microbiol Ecol 2007, 62:142-160.

21. Sekirov I, Russell SL, Antunes LC, Finlay BB: Gut microbiota in health and disease. Physiol Rev 2010, 90:859-904.

22. Griffiths D, Fawley W, Kachrimanidou M, Bowden R, Crook DW, Fung R, Golubchik T, Harding RM, Jeffery KJ, Jolley KA, Kirton R, Peto TE, Rees G, Stoesser N, Vaughan A, Walker AS, Young BC, Wilcox M, Dingle KE: Multilocus sequence typing of Clostridium difficile. J Clin Microbiol 2010, 48:770-778.

23. Manges AR, Labbe A, Loo VG, Atherton JK, Behr MA, Masson L, Tellis PA, Brousseau R: Comparative metagenomic study of alterations to the intestinal microbiota and risk of nosocomial Clostridum difficileassociated disease. J Infect Dis 2010, 202:1877-1884.

24. Petrof EO, Gloor GB, Vanner SJ, Weese SJ, Carter D, Daigneault MC, Brown EM, Schroeter K, Allen-Vercoe E: Stool substitute transplant therapy for the eradication of Clostridium difficile infection: 'RePOOPulating' the gut. Microbiome 2013, 1:3.

25. Shahinas D, Silverman M, Sittler T, Chiu C, Kim P, Allen-Vercoe E, Weese S, Wong A, Low DE, Pillai DR: Toward an understanding of changes in diversity associated with fecal microbiome transplantation based on $16 \mathrm{~S}$ rRNA gene deep sequencing. mBio 2012. doi:10.1128/mBio.00338-12.

26. Ananthakrishnan AN: Clostridium difficile infection: epidemiology, risk factors and management. Nat Rev Gastroenterol Hepatol 2011, 8:17-26. 
27. Willing BP, Dicksved J, Halfvarson J, Andersson AF, Lucio M, Zheng Z, Jarnerot G, Tysk C, Jansson JK, Engstrand L: A pyrosequencing study in twins shows that gastrointestinal microbial profiles vary with inflammatory bowel disease phenotypes. Gastroenterology 2010, 139:1844-1854. e1841.

28. Booijink CC, El-Aidy S, Rajlic-Stojanovic M, Heilig HG, Troost FJ, Smidt H, Kleerebezem M, De Vos WM, Zoetendal EG: High temporal and inter-individual variation detected in the human ileal microbiota. Environ Microbiol 2010, 12:3213-3227.

29. van den Bogert B, de Vos WM, Zoetendal EG, Kleerebezem M: Microarray analysis and barcoded pyrosequencing provide consistent microbial profiles depending on the source of human intestinal samples. Appl Environ Microbiol 2011, 77:2071-2080.

30. Zoetendal EG, Raes J, van den Bogert B, Arumugam M, Booijink CC, Troost FJ, Bork P, Wels M, de Vos WM, Kleerebezem M: The human small intestinal microbiota is driven by rapid uptake and conversion of simple carbohydrates. ISME J 2012, 6:1415-1426.

31. Anderson CM, Langford RF: Bacterial content of small intestine of children in health, in coeliac disease, and in fibrocystic disease of pancreas. Br Med J 1958, 1:803-806.

32. Reuter $\mathrm{G}$ : The Lactobacillus and Bifidobacterium microflora of the human intestine: composition and succession. Curr Issues Intest Microbiol 2001, 2:43-53.

33. Sullivan A, Tornblom H, Lindberg G, Hammarlund B, Palmgren AC, Einarsson C, Nord CE: The micro-flora of the small bowel in health and disease. Anaerobe 2003, 9:11-14.

34. Morgan XC, Tickle TL, Sokol H, Gevers D, Devaney KL, Ward DV, Reyes JA, Shah SA, LeLeiko N, Snapper SB, Bousvaros A, Korzenik J, Sands BE, Xavier $R J$, Huttenhower C: Dysfunction of the intestinal microbiome in inflammatory bowel disease and treatment. Genome Biol 2012, 13:R79.

35. Antharam VC, Li EC, Ishmael A, Sharma A, Mai V, Rand KH, Wang GP: Intestinal dysbiosis and depletion of butyrogenic bacteria in Clostridium difficile infection and nosocomial diarrhea. J Clin Microbiol 2013, 51:2884-2892.

36. Meihoff WE, Kern F Jr: Bile salt malabsorption in regional ileitis, ileal resection and mannitol-induced diarrhea. J Clin Invest 1968, 47:261-267.

37. Swidsinski A, Loening-Baucke V, Vaneechoutte M, Doerffel Y: Active Crohn's disease and ulcerative colitis can be specifically diagnosed and monitored based on the biostructure of the fecal flora. Inflamm Bowel Dis 2008, 14:147-161.

38. Manichanh C, Reeder J, Gibert P, Varela E, Llopis M, Antolin M, Guigo R, Knight R, Guarner F: Reshaping the gut microbiome with bacterial transplantation and antibiotic intake. Genome Res 2010, 20:1411-1419.

39. Espey MG: Role of oxygen gradients in shaping redox relationships between the human intestine and its microbiota. Free Radic Biol Med 2013, 55:130-140.

40. Jarchum I, Liu M, Shi C, Equinda M, Pamer EG: Critical role for MyD88mediated neutrophil recruitment during Clostridium difficile colitis. Infect Immun 2012, 80:2989-2996.

41. Samuelson J: Why metronidazole is active against both bacteria and parasites. Antimicrob Agents Chemother 1999, 43:1533-1541.

42. Watanakunakorn C: Mode of action and in-vitro activity of vancomycin. J Antimicrob Chemother 1984(Suppl D):7-18.

43. Caporaso JG, Lauber CL, Costello EK, Berg-Lyons D, Gonzalez A, Stombaugh J, Knights D, Gajer P, Ravel J, Fierer N, Gordon JI, Knight R: Moving pictures of the human microbiome. Genome Biol 2011, 12:R50.

44. Song Y, Garg S, Girotra M, Maddox C, von Rosenvinge EC, Dutta A, Dutta S, Fricke WF: Microbiota dynamics in patients treated with fecal microbiota transplantation for recurrent Clostridium difficile infection. PLOS ONE 2013, 8:e81330.

45. Landy J, Al-Hassi HO, McLaughlin SD, Walker AW, Ciclitira PJ, Nicholls RJ, Clark SK, Hart AL: Review article: faecal transplantation therapy for gastrointestinal disease. Aliment Pharmacol Ther 2011, 34:409-415.
46. Guo B, Harstall C, Louie T, Veldhuyzen Van Zanten S, Dieleman LA: Systematic review: faecal transplantation for the treatment of Clostridium difficile-associated disease. Aliment Pharmacol Ther 2012, 35:865-875.

47. Borody TJ, Khoruts A: Fecal microbiota transplantation and emerging applications. Nat Rev Gastroenterol Hepatol 2011, 9:88-96.

48. Aroniadis OC, Brandt LJ: Fecal microbiota transplantation: past, present and future. Curr Opin Gastroenterol 2012, 29:79-84.

doi:10.1186/2049-2618-2-13

Cite this article as: Shankar et al: Species and genus level resolution analysis of gut microbiota in Clostridium difficile patients following fecal microbiota transplantation. Microbiome 2014 2:13.

\section{Submit your next manuscript to BioMed Central and take full advantage of:}

- Convenient online submission

- Thorough peer review

- No space constraints or color figure charges

- Immediate publication on acceptance

- Inclusion in PubMed, CAS, Scopus and Google Scholar

- Research which is freely available for redistribution

Submit your manuscript at www.biomedcentral.com/submit

C) Biomed Central 